\title{
ON THE RADICAL OF THE MODULE CATEGORY OF AN ENDOMORPHISM ALGEBRA
}

\author{
CLAUDIA CHAIO AND VICTORIA GUAZZELLI
}

\begin{abstract}
Given a finite dimensional algebra $A$ over an algebraically closed field we study the relationship between the powers of the radical of a morphism in the module category of the algebra $A$ and the induced morphism in the module category of the endomorphism algebra of a tilting $A$-module. We compare the nilpotency indices of the radical of the mentioned module categories. We find an upper bound for the nilpotency index of the radical of the module category of iterated tilted algebras of Dynkin type.
\end{abstract}

\section{INTRODUCTION}

Let $A$ be an a finite dimensional $k$-algebra over an algebraically closed field $k$, and $\bmod A$ the category of finitely generated left $A$-modules. For $X, Y \in \bmod A$, we denote by $\Re(X, Y)$ the set of all morphisms $f: X \rightarrow Y$ such that, for all indecomposable $A$-module $M$, each pair of morphisms $h: M \rightarrow X$ and $h^{\prime}: Y \rightarrow M$ the composition $h^{\prime} f h$ is not an isomorphism. Inductively, the powers of $\Re(X, Y)$ are defined. By $\Re_{A}^{\infty}(X, Y)$ we denote the intersection of all powers $\Re_{A}^{i}(X, Y)$ of $\Re_{A}(X, Y)$, with $i \geq 1$.

An important research direction towards understanding the structure of a module category is the study of the compositions of irreducible morphisms in relation with the powers of the radical of their module categories, see for example [9], [10] and [13.

In case we deal with a representation-finite algebra, it is well-known that there is a positive integer $n$ such that $\Re^{n}(\bmod A)=0$, see [3, p. 183]. The minimal lower bound $m \geq 1$ such that $\Re^{m}(\bmod A)$ vanishes is called the nilpotency index of $\Re(\bmod A)$.

In [8], the first named author determined the nilpotency index of $\Re(\bmod A)$ in terms of the left and right degrees of same particular irreducible morphisms. The notion of degree of an irreducible morphisms was introduced by $\mathrm{S}$. Liu in [16]. In this work, we improve such a result. More precisely, we reduce the steps to compute such a bound in case we deal with algebras such that all vertices in the ordinary quiver are neither sinks nor sources, see Theorem 2.16.

One of the aims of this article is to study the relationship between the power of the radical of the morphisms in $\bmod A$ and the induced morphisms in $\bmod B$, where $B$ is the endomorphism algebra of some tilting $A$-modules. To achieve to this result, we strongly use the well-known tilting theorem due to Brenner and Butler.

We also compare the nilpotency indices of the radical of the module category of an algebra and of the endomorphism algebra of some specific tilting $A$-modules. Precisely, we prove the following result.

Theorem A. Let $A \simeq k Q_{A} / I_{A}$ be a representation-finite algebra, $T$ be a separating tilting $A$-module and $B=\operatorname{End}_{A} T$. Consider $\left(R_{A}\right)_{0}$ to be the set of all the vertices $u \in Q_{A}$ such that the length of any path from $P_{u}$ to $I_{u}$ going through $S_{u}$ is $r_{A}-1$, where $r_{A}$ is the nilpotency index

2000 Mathematics Subject Classification. 16G70, 16G20, 16E10.

Key words and phrases. Irreducible morphisms, Degrees, Radical, tilting module. 
of $\Re(\bmod A)$ and $P_{u}, I_{u}$ and $S_{u}$ are the projective, the injective and the simple corresponding to the vertex $u \in Q_{A}$, respectively. If $B$ is representation-finite and for some $u \in\left(R_{A}\right)_{0}$ we have that $P_{u} \in \operatorname{add} T$ then $r_{A} \leq r_{B}$, where $r_{B}$ is the nilpotency index of $\Re(\bmod B)$.

As a consequence we obtain information between the nilpotency index of the radical of the module category of an algebra and of the endomorphism algebra of an APR-tilting module, when both algebras are of finite representation type.

In [18], D. Zacharia determined the nilpotency index of the radical of the module category of any representation-finite hereditary algebra in terms of the number of vertices of their ordinary quiver.

For an iterated tilted algebra of Dynkin type, we find an upper bound of such an index. In many cases, this bound coincide with the nilpotency index, see Example 3.7. More precisely, we prove Theorem B.

Theorem B. Let $\Delta$ be a Dynkin quiver and $A$ be an iterated tilted algebra of type $\Delta$. Consider $r_{A}$ the nilpotency index of $\Re_{A}(\bmod A)$ and $\bar{\Delta}$ the underlying graph.

(a) If $\bar{\Delta}=A_{n}$, then $r_{A} \leq n$ for $n \geq 1$.

(b) If $\bar{\Delta}=D_{n}$, then $r_{A} \leq 2 n-3$ for $n \geq 4$.

(c) If $\bar{\Delta}=E_{6}$, then $r_{A} \leq 11$.

(d) If $\bar{\Delta}=E_{7}$, then $r_{A} \leq 17$.

(e) If $\bar{\Delta}=E_{8}$, then $r_{A} \leq 29$.

This paper is organized as follows. In the first section, we state some notations and recall some preliminaries results. Section 2 is dedicated to prove the results concerning the comparison between morphisms in $\bmod A$ and the induced morphisms in $\bmod B$, in relation with the power of the radical of their module categories. We prove Theorem A. In Section 3, we apply the results concerning the nilpotency index of the radical of the module category of a representation-finite hereditary algebra to find an upper bound for nilpotency index of the radical of the module category of a iterated tilted algebras of Dynkin type. Precisely, we prove Theorem B.

Both authors thankfully acknowledge partial support from CONICET and from Universidad Nacional de Mar del Plata, Argentina. The first author is a researcher from CONICET.

\section{PRELIMINARIES}

Throughout this work, by an algebra we mean a finite dimensional basic $k$-algebra over an algebraically closed field, $k$.

1.1. A quiver $Q$ is given by a set of vertices $Q_{0}$ and a set of arrows $Q_{1}$, together with two maps $s, e: Q_{1} \rightarrow Q_{0}$. Given an arrow $\alpha \in Q_{1}$, we write $s(\alpha)$ the starting vertex of $\alpha$ and $e(\alpha)$ the ending vertex of $\alpha$. If $A$ is an algebra then there exists a quiver $Q_{A}$, called the ordinary quiver of $A$, such that $A$ is the quotient of the path algebra $k Q_{A}$ by an admissible ideal.

1.2. Let $A$ be an algebra. We denote by $\bmod A$ the category of finitely generated left $A$ modules and by ind $A$ the full subcategory of $\bmod A$ which consists of one representative of each isomorphism class of indecomposable $A$-modules.

For any $A$-module $M$, we denote by add $M$ the full subcategory of $\bmod A$ whose objects are the direct sums of summands of $M$.

We denote by $\Gamma_{A}$ the Auslander-Reiten quiver of $\bmod A$, by $\tau$ and $\tau^{-1}$ the AuslanderReiten translation DTr and $\operatorname{TrD}$, respectively. 
1.3. Consider $X, Y \in \bmod A$. The ideal $\Re(X, Y)$ is the set of all the morphisms $f: X \rightarrow Y$ such that, for each $M \in$ ind $A$, each $h: M \rightarrow X$ and each $h^{\prime}: Y \rightarrow M$ the composition $h^{\prime} f h$ is not an isomorphism. For $n \geq 2$, the powers of $\Re(X, Y)$ are defined inductively. By $\Re^{\infty}(X, Y)$ we denote the intersection of all powers $\Re^{i}(X, Y)$ of $\Re(X, Y)$, with $i \geq 1$.

Following [11], we say that the depth of a morphism $f: M \rightarrow N$ in $\bmod A$ is infinite if $f \in \Re^{\infty}(M, N)$; otherwise, the depth of $f$ is the integer $n \geq 0$ for which $f \in \Re^{n}(M, N)$ but $f \notin \Re^{n+1}(M, N)$. We denote the depth of $f$ by $\operatorname{dp}(f)$.

A morphism $f: X \rightarrow Y$, with $X, Y \in \bmod A$, is called irreducible provided it does not split and whenever $f=g h$, then either $h$ is a split monomorphism or $g$ is a split epimorphism.

By [4], it is known that a morphism $f: X \rightarrow Y$, with $X, Y \in$ ind $A$, is irreducible if and only if $\operatorname{dp}(f)=1$.

Next, we recall the definition of degree of an irreducible morphism given by S. Liu in [16], a notion fundamental for this work.

Let $f: X \rightarrow Y$ be an irreducible morphism in $\bmod A$, with $X$ or $Y$ indecomposable. The left degree $d_{l}(f)$ of $f$ is infinite, if for each integer $n \geq 1$, each module $Z \in$ ind $A$ and each morphism $g: Z \rightarrow X$ with $\operatorname{dp}(g)=n$ we have that $f g \notin \Re^{n+2}(Z, Y)$. Otherwise, the left degree of $f$ is the least natural $m$ such that there is an $A$-module $Z$ and a morphism $g: Z \rightarrow X$ with $\operatorname{dp}(g)=m$ such that $f g \in \Re^{m+2}(Z, Y)$.

The right degree $d_{r}(f)$ of an irreducible morphism $f$ is dually defined.

We say that an algebra $\mathrm{A}$ is representation-finite if there is only a finite number of isomorphisms classes of indecomposable A-modules. It is well-known that an algebra $A$ is representation-finite if and only if there is a positive integer $n$ such that $\Re^{n}(X, Y)=0$ for each $A$-module $X$ and $Y$. The minimal lower bound $m$ such that $\Re^{m}(\bmod A)=0$ is called the nilpotency index of $\Re(\bmod A)$. We denote it by $r_{A}$.

If $A$ is representation-finite, by [10] we know that all irreducible epimorphisms and all irreducible monomorphisms are of finite left and right degree, respectively. We denote by $P_{a}$, $I_{a}$ and $S_{a}$ the projective, the injective and the simple $A$-module corresponding to the vertex $a$ in $Q_{A}$, respectively.

In [8], the first named author, find the nilpotency index of a representation-finite algebra in terms of degrees of some irreducible morphisms and also in terms of length of paths. Precisely, the author proved the following result, fundamental for our purposes.

If either $P_{a}=S_{a}$ or $I_{a}=S_{a}$ then we write $n_{a}=0$ or $m_{a}=0$, respectively. Otherwise, we consider the irreducible morphisms $\iota_{a}: \operatorname{rad}\left(P_{a}\right) \hookrightarrow P_{a}$ and $g_{a}: I_{a} \longrightarrow I_{a} / \operatorname{soc}\left(I_{a}\right)$ and we write $n_{a}=d_{r}\left(\iota_{a}\right)$ and $m_{a}=d_{l}\left(g_{a}\right)$. We write $r_{a}=n_{a}+m_{a}$.

Theorem 1.4. ([8, Theorem A].) Let $A \simeq k Q_{A} / I_{A}$ be a finite dimensional algebra over an algebraically closed field and assume that $A$ is of finite representation type. Then, the nilpotency index $r_{A}$ of the radical of the module category of $\bmod A$ is $\max \left\{r_{a}\right\}_{a \in Q_{0}}+1$.

A path in $\bmod A$ is a sequence of non-zero non-isomorphisms between indecomposable $A$ modules. In case that the morphisms involved in the above path are irreducible, we say that it is a path in ind $A$.

The length of a path $M_{0} \stackrel{f_{1}}{\rightarrow} M_{1} \stackrel{f_{2}}{\rightarrow} M_{2} \rightarrow \ldots \rightarrow M_{t-1} \stackrel{f_{t}}{\rightarrow} M_{t}$, between indecomposable $A$-modules is the number $t$ of irreducible morphisms in it. Given $M, N \in$ ind $A$, we denote a path in ind $A$ from $M$ to $N$ by the expression $M \rightsquigarrow N$.

By [8, Remark 1], we know that $r_{a}$ is equal to the length of any path of irreducible morphisms between indecomposable modules from the projective $P_{a}$ to the injective $I_{a}$, going through the simple $S_{a}$. Therefore, in order to find the nilpotency index we have to find the path of maximum length between them. 
For the convenience of the reader we recall a result from [8] that we shall use frequently in this work.

Lemma 1.5. [8, Lema 2.4] Let $A \cong k Q / I$ be a representation-finite algebra. Given a $\in Q_{0}$, consider $r_{a}$ the number defined above. Then,

(a) every nonzero morphism $f: P_{a} \rightarrow I_{a}$ that factors through the simple A-module $S_{a}$ is such that $d p(f)=r_{a}$.

(b) every non-zero morphism $f: P_{a} \rightarrow I_{a}$ which does not factor through the simple Amodule $S_{a}$ is such that $d p(f)=k$, with $0 \leq k<r_{a}$.

1.6. Let $\Gamma$ be a component of $\Gamma_{A}$. Following [12, we say that $\Gamma$ is a component with length if parallel paths in $\Gamma$ have the same length. By parallel paths we mean paths in ind $A$ having the same starting vertex and the same ending vertex.

Given a directed component $\Gamma$ of $\Gamma_{A}$, its orbit graph, denoted by $O(\Gamma)$, has as points the $\tau$-orbits $O(M)$ of the modules $\mathrm{M}$ in $\Gamma$. There exists an edge between $O(M)$ and $O(N)$ in $O(\Gamma)$ if there are integer numbers $m$ and $n$ and an irreducible morphism $\tau^{m} M \rightarrow \tau^{n} N$ or $\tau^{n} N \rightarrow \tau^{m} M$. Recall that if the orbit graph $O(\Gamma)$ is of tree-type, then $\Gamma$ is a simply connected translation quiver, and therefore, by [6], $\Gamma$ is a component with length.

For general references on radical theory we refer the reader to [3].

1.7. A pair $(\mathcal{T}, \mathcal{F})$ of full subcategories of $\bmod A$ is called a torsion theory if the following conditions are satisfied:

(a) $\operatorname{Hom}_{A}(M, N)=0$ for all $M \in \mathcal{T}$ y $N \in \mathcal{F}$.

(b) If $\operatorname{Hom}_{A}(M, F)=0$ for all $F \in \mathcal{F}$, implies $M \in \mathcal{T}$.

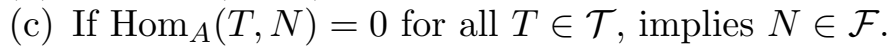

According to [15], an A-module $T$ is called a tilting module if $\operatorname{pd} T \leq 1, \operatorname{Ext}_{A}^{1}(T, T)=0$ and the number of isomorphism classes of indecomposable summands of $T$ equals the number of vertices in $Q_{A}$.

Given $T$ a tilting $A$-module, there exists a close connection between the representation theories of $A$ and of $B=\operatorname{End}_{A}(T)$. It is well-known that a tilting $A$-module induces a torsion pair $(\mathcal{T}(T), \mathcal{F}(T))$ in $\bmod A$ and a torsion pair $(\mathcal{X}(T), \mathcal{Y}(T))$ in $\bmod B$. More precisely, it was shown in [5] and [15] that if $T$ is a tilting module and $B=\operatorname{End}_{A}(T)$, then $T$ is a tilting $B$ module and $A \simeq \operatorname{End}_{B} T$ and the functors $\operatorname{Hom}_{A}(T,-)$ and $-\otimes_{B} T$ induce inverse equivalences between the full subcategories $\mathcal{T}(T)=\left\{X: \operatorname{Ext}_{A}^{1}(T, X)=0\right\}$ and $\mathcal{Y}(T)=\left\{Y: \operatorname{Tor}_{1}^{B}(Y ; T)=\right.$ $0\}$, while the functors $\operatorname{Ext}_{A}^{1}(T,-)$ and $\operatorname{Tor}_{1}^{B}(-, T)$ induce inverse equivalences between the full subcategories $\mathcal{F}(T)=\left\{X: \operatorname{Hom}_{A}(T, X)=0\right\}$ and $\mathcal{X}(T)=\left\{Y: Y \otimes_{B} T=0\right\}$. This last result is well-known as Brenner and Butler's theorem.

A tilting $A$-module $T$ is called separating (splitting, respectively) if, for each indecomposable $A$-module $M(B$-module $N$, respectively), we have either $M \in \mathcal{T}(T)$ or $M \in \mathcal{F}(T)$ $(N \in \mathcal{X}(T)$ or $N \in \mathcal{Y}(T)$, respectively).

An example, of a separating tilting $A$ module, is provided by the so-called APR-tilting modules. Let $P_{a}$ be a simple projective non-injective module (corresponding to the sink $\left.a \in Q_{0}\right)$. Then, the module $T[a]=\tau^{-1}\left(S_{a}\right) \oplus\left(\oplus_{b \neq a} P_{b}\right)$ is called the APR-tilting module associated to the vertex $a$. Moreover, $\mathcal{F}(T[a])=\operatorname{add} S_{a}$ and $\mathcal{T}(T[a])=\operatorname{add}\left(\operatorname{ind} A \backslash S_{a}\right)$.

In [1, the author proved that an APR-tilting $A$-module $T[a]$ is splitting if and only if the vertex $a$ is a free sink, that is, $a$ is not an end point of a zero relation on $Q_{A}$. Moreover, the vertex of $Q_{B}$ corresponding to $a$ is a source.

For general references on tilting theory we refer the reader to [1], [2] and [15]. 
1.8. Consider $H \cong k Q_{H}$ a representation-finite hereditary algebra, that is, $Q_{H}$ is a Dynkin quiver. We end up this section recalling the definition of an iterated tilting algebra of type $\Delta$.

Consider $\Delta$ a finite connected quiver without oriented cycles. An algebra $A$ is called an iterated tilting algebra of type $\Delta$ if there exist a sequence of algebras $A=A_{0}, A_{1}, \ldots, A_{m}=k \Delta$ and a sequence of separating $A_{i}$-tilting modules $T^{(i)}$, for $0 \leq i<m$, such that $A_{i+1}=$ End $_{A_{i}} T^{(i)}$. In particular, if $m=1$ then $A$ is called a tilted algebra.

\section{ON THE NILPOTENCY INDEX}

Throughout this work, by $r_{A}$ we denote the nilpotency index of the radical of the module category of a representation-finite algebra and by $r_{B}$ the nilpotency index of the radical of the module category of the endomorphism algebra of a tilting $A$-module.

The aim of this section is to compare the nilpotency indices of the radical of the module category of a given algebra $A$ and the module category of the endomorphism algebra of a tilting $A$-module.

First, we establish the relationship between the powers of the radical of a morphism in the module category of $A$ and the induced morphism in the module category of the endomorphism algebra of a tilting $A$-module.

In the next result, we study the situation when $T$ is a separating tilting module. A dual result holds for a splitting tilting module.

Proposition 2.1. Let $A$ be an algebra, $T$ be a separating tilting $A$-module and $B=\operatorname{End}_{A}(T)$. Let $M, N$ be indecomposable $A$-modules in $\mathcal{T}(T)$. If $f \in \Re_{A}^{n}(M, N)$ for some $n \geq 1$ then $F(f) \in \Re_{B}^{n}(F(M), F(N))$, where $F=\operatorname{Hom}_{A}(T,-)$.

In addition, if $d p(F(f))=n$ then there is a non-zero path of irreducible morphisms between indecomposable $B$-modules in $\mathcal{Y}(T)$ from $F(M)$ to $F(N)$ of length $n$.

Proof. Let $M, N$ be indecomposable $A$-modules in $\mathcal{T}(T)$ and $f \in \Re_{A}^{n}(M, N)$, for some $n \geq 1$. We prove by induction on $n$ that $F(f) \in \Re_{B}^{n}(F(M), F(N))$.

If $n=1$ then $f$ is not an isomorphism. Hence, by Brenner and Butler's theorem it follows that $F(f)$ is not an isomorphism in $\bmod B$. Therefore, $F(f) \in \Re_{B}(F(M), F(N))$.

Now, consider $n \geq 2$. By [3, Proposition 7.4], there exists a positive integer $s \geq 1$ and indecomposable $A$-modules $X_{1}, \ldots, X_{s}$ such that $f=\Sigma_{i=1}^{s} g_{i} f_{i}$, with $f_{i} \in \Re_{A}\left(M, X_{i}\right)$ and $g_{i}=\Sigma_{k=1}^{t_{i}} h_{i}^{k}$, where each $h_{i}^{k}$ is a composition of $n-1$ irreducible morphisms between indecomposable $A$-modules, for $1 \leq i \leq s, 1 \leq k \leq t_{i}$ and $1 \leq j \leq n$. Therefore, $g_{i} \in \Re_{A}^{n-1}\left(X_{i}, N\right)$ for $1 \leq i \leq s$. Without loss of generality, since $f \neq 0$, we may assume that for all $i$, the morphisms $f_{i}$ and $g_{i}$ do not vanish.

Since $T$ is a separating tilting $A$-module, then all the indecomposable modules $X_{i}$, with $1 \leq i \leq s$, belong to $\mathcal{T}(T)$ because $M \in \mathcal{T}(T)$ and $\operatorname{Hom}(\mathcal{T}(T), \mathcal{F}(T))=0$. Hence, by Brenner and Butler's theorem we have that $F(f)=\sum_{i=1}^{s} F\left(g_{i}\right) F\left(f_{i}\right)$, with $F\left(f_{i}\right) \in$ $\Re_{A}\left(F(M), F\left(X_{i}\right)\right)$. Then, by inductive hypothesis $F\left(g_{i}\right) \in \Re_{B}^{n-1}\left(F\left(X_{i}\right), F(N)\right)$. Therefore $F(f) \in \Re_{B}^{n}(F(M), F(N))$.

Now, assume that $F(f) \notin \Re_{B}^{n+1}(F(M), F(N))$. Thus $f \notin \Re_{A}^{n+1}(M, N)$. If $n=1$ then $F(f)$ is irreducible and there is a path from $F(M)$ to $F(N)$ of length one between modules in $\mathcal{Y}(T)$.

If $n \geq 2$, since $d p(f)=n$, then by [3, Proposition 7.4], there exists a positive integer $s \geq 1$ such that $f=\Sigma_{i=1}^{s} \Sigma_{k=1}^{t_{i}} h_{i}^{k} f_{i}$, where each $h_{i}^{k}=h_{i}^{k, n-1} \ldots h_{i}^{k, 1}$ is a composition of $n-1$ 
irreducible morphisms between indecomposable modules in $\mathcal{T}(T)$. Then,

$$
F(f)=\Sigma_{i=1}^{s} \Sigma_{k=1}^{t_{i}} F\left(h_{i}^{k, n-1}\right) \ldots F\left(h_{i}^{k, 1}\right) F\left(f_{i}\right) .
$$

If all the summands of $F(f)$ belong to $\Re_{B}^{n+1}(F(M), F(N))$, then $F(f) \in \Re_{B}^{n+1}(F(M), F(N))$, getting a contradiction to our assumption. Hence, there exist at least an $i_{0} \in\{1, \ldots, s\}$ and a $k_{0} \in\left\{1, \ldots, t_{i_{0}}\right\}$ such that $d p\left(F\left(h_{i_{0}}^{k_{0}, n-1}\right) \ldots F\left(h_{i_{0}}^{k_{0}, 1}\right) F\left(f_{i_{0}}\right)\right)=n$. Thus, it is a composition of $n$ irreducible morphisms between indecomposable $B$-modules in $\mathcal{Y}(T)$, proving the result.

The above result holds if we consider a morphism between indecomposable modules in the free-torsion class. Bellow, we state the result.

Proposition 2.2. Let $A$ be an algebra, $T$ be a separating tilting $A$-module and $B=\operatorname{End}_{A}(T)$. Let $M, N$ be indecomposable $A$-modules in $\mathcal{F}(T)$. If $g \in \Re_{A}^{n}(M, N)$ for some $n \geq 1$ then $F^{\prime}(g) \in \Re_{B}^{n}\left(F^{\prime}(M), F^{\prime}(N)\right)$, where $F^{\prime}=\operatorname{Ext}_{A}^{1}(T,-)$.

In addition, if $d p\left(F^{\prime}(g)\right)=n$ then there is a non-zero path of irreducible morphisms between indecomposable $B$-modules in $\mathcal{X}(T)$ from $F^{\prime}(M)$ to $F^{\prime}(N)$ of length $n$.

Our next examples show that the above result can not be improved taking into account the depth of the morphism.

Example 2.3. (a) First, we show an example of a morphism which does not belong to $\Re_{A}^{2}$ but where the induced morphism is in $\Re_{B}^{2}$.

Let $A$ be an algebra given by the quiver

$$
1 \stackrel{\alpha}{\longrightarrow} 2 \stackrel{\beta}{\longrightarrow} 3 \stackrel{\gamma}{\longrightarrow} 4
$$

with $I_{A}=<\gamma \beta \alpha>$. The Auslander-Reiten quiver of $\bmod A$ is the following:

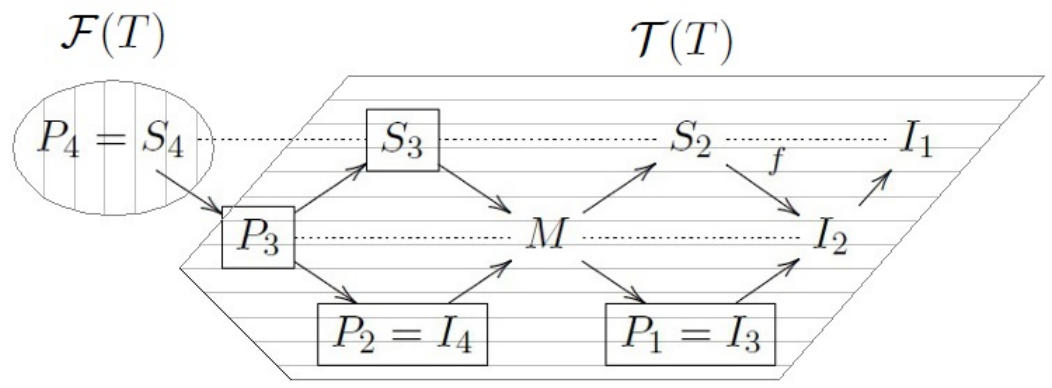

where $T=P_{1} \oplus P_{2} \oplus P_{3} \oplus S_{3}$ is a tilting $A$-module. The endomorphism algebra $B=\operatorname{End}_{A} T$ is given by the bound quiver:

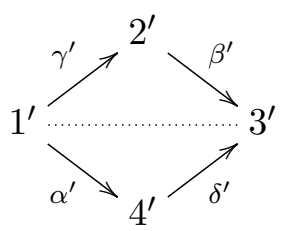

with $I_{B}=<\beta^{\prime} \gamma^{\prime}-\delta^{\prime} \alpha^{\prime}>$. The Auslander-Reiten quiver of $\bmod B$ is the following 


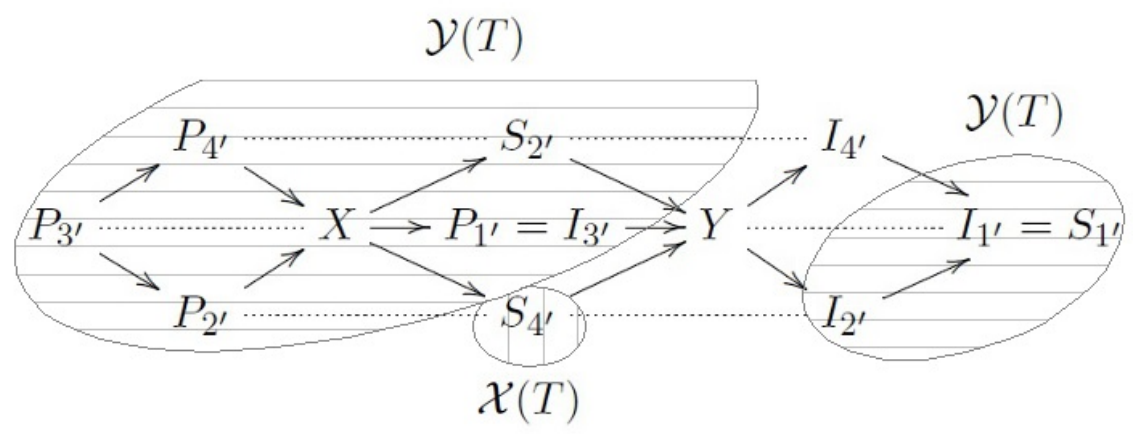

Observe that $T$ is a separating but not splitting tilting $A$-module. The morphism $f: S_{2} \rightarrow I_{2}$ does not belong to $\Re_{A}^{2}\left(S_{2}, I_{2}\right)$, while the induced morphism $\operatorname{Hom}_{A}(T, f)$ is in $\Re_{B}^{2}\left(S_{2^{\prime}}, I_{2^{\prime}}\right)$.

(b) Our next example shows a morphism in $\bmod A$ such that $d p(f)=t$ and where the induced morphism belongs to $\Re_{B}^{\infty}$.

Let $A$ be the algebra given by the quiver

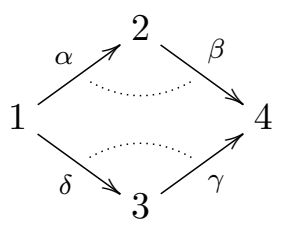

with $I_{A}=<\beta \alpha, \gamma \delta>$. The Auslander-Reiten quiver of $\bmod A$ is the following:

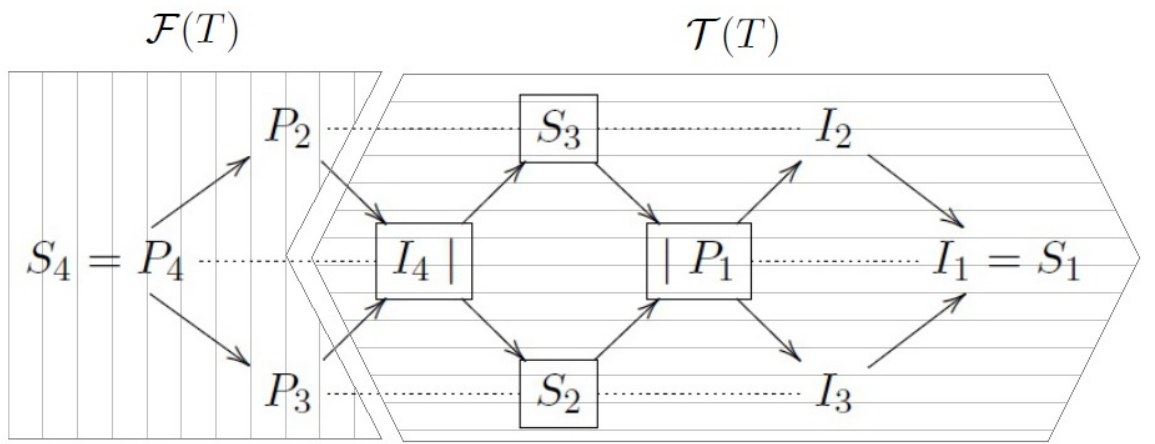

Consider the tilting module $T=I_{4} \oplus S_{3} \oplus S_{2} \oplus P_{1}$. The endomorphisms algebra $B=\operatorname{End} T$ is given by the quiver

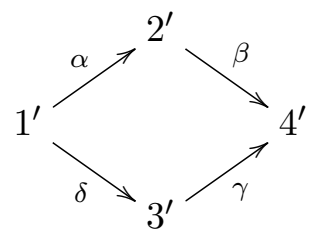

Note that $B$ is a hereditary algebra of type $\widetilde{A}_{3}$. Consider the morphism $f: P_{1} \rightarrow I_{1}$ in $\bmod A$. By 1.5 we know that $\operatorname{dp}(f)=2$. On the other hand, if we applied to $f$ the functor $F=\operatorname{Hom}_{A}(T,-)$, the morphism $F(f)$ belongs to $\Re_{B}^{\infty}\left(F\left(P_{1}\right), F\left(I_{1}\right)\right)$, since $F\left(P_{1}\right)$ and 
$F\left(I_{1}\right)$ is a projective and an injective $B$-module, respectively, in different Auslander-Reiten components of $\bmod B$.

As a consequence of Proposition 2.1 and Proposition 2.2, we deduce the following corollary.

Corollary 2.4. Let $A$ be an algebra, $T$ be a separating and splitting tilting $A$-module and $B=$ $\operatorname{End}_{A}(T)$. Let $M, N$ be indecomposable $A$-modules in $\mathcal{T}(T)$ (or in $\mathcal{F}(T)$ ). Then, for some $n \geq$ $1, f \in \Re_{A}^{n}(M, N)$ if and only if $F(f) \in \Re_{B}^{n}(F(M), F(N))$ (or $\left.F^{\prime}(f) \in \Re_{B}^{n}\left(F^{\prime}(M), F^{\prime}(N)\right)\right)$, where $F=\operatorname{Hom}_{A}(T,-)$ (or $\left.F^{\prime}=\operatorname{Ext}_{A}^{1}(T,-)\right)$.

Next, we show an example where the above results do not hold if we consider a morphism $f: M \rightarrow N$, where the domain and codomain belong to different subcategories.

Example 2.5. Let $A$ be the algebra given by the quiver

$$
1 \longrightarrow 2 \longrightarrow 3 \longrightarrow 4
$$

The Auslander-Reiten quiver of $\bmod A$ is the following:

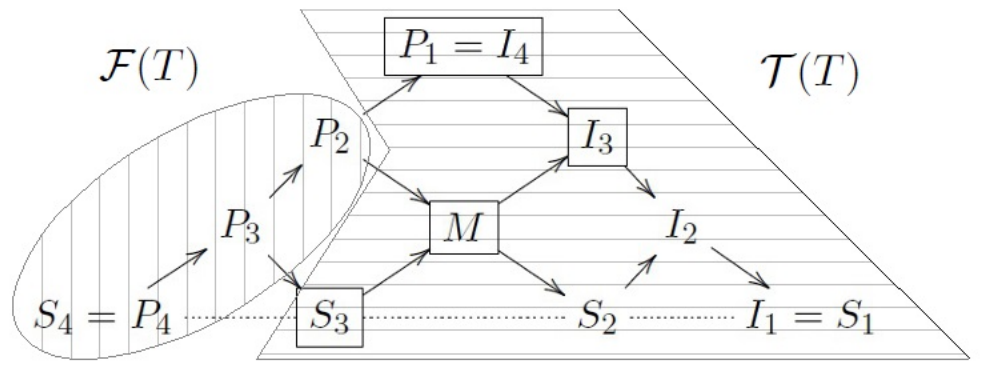

where $T=P_{1} \oplus I_{3} \oplus M \oplus S_{3}$ is a tilting $A$-module. The endomorphism algebra $B=\operatorname{End}_{A} T$ is given by the quiver:

$$
1^{\prime}<2^{\prime} \longrightarrow 3^{\prime} \longrightarrow 4^{\prime}
$$

and the Auslander-Reiten quiver of $\bmod B$ is the following

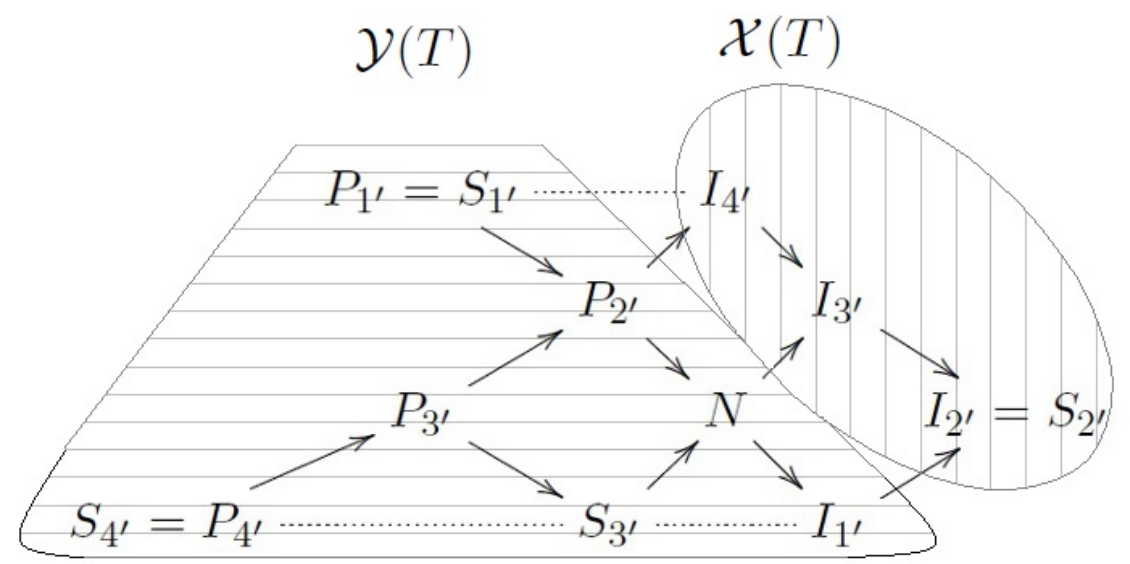


Now, if we consider an irreducible morphism $f: P_{2} \rightarrow P_{1}$ in $\bmod A$, where $P_{2} \in \mathcal{F}(T)$ and $P_{1} \in \mathcal{T}(T)$, we have that $d p(f)=1$.

On the other hand, $\operatorname{Ext}_{A}^{1}\left(T, P_{2}\right) \simeq S_{2^{\prime}}$ and $\operatorname{Hom}_{A}\left(T, P_{1}\right) \simeq S_{1^{\prime}}$ are non-isomorphic simple $B$-modules, and the unique morphism between them is the zero morphism, which belongs to the infinite radical.

As an immediate consequence of Corollary 2.4, we get the following result.

Corollary 2.6. Let $A$ be an algebra, $T$ be a separating and splitting tilting $A$-module and $B=$ $\operatorname{End}_{A}(T)$. Then, $f: M \rightarrow N$ is an irreducible morphism between indecomposable A-modules in $\mathcal{T}(T)(\mathcal{F}(T)$, respect. $)$ if and only if $F(f): F(M) \rightarrow F(N)\left(F^{\prime}(f): F^{\prime}(M) \rightarrow F^{\prime}(N)\right.$, respect.) is an irreducible morphism between indecomposable $B$-modules in $\mathcal{Y}(T)(\mathcal{X}(T)$, respect.), where $F=\operatorname{Hom}_{A}(T,-)\left(F^{\prime}=\operatorname{Ext}_{A}^{1}(T,-)\right.$, respect. $)$.

Our next aim is to compare the nilpotency indices of the radical of the module category of an algebra and the module category of the endomorphism algebra of some particular tilting modules.

For any algebra $A$ it is well-known that if $T$ is a separating tilting $A$-module and $B=$ $\operatorname{End}_{A}(T)$ is representation-finite, then so is $A$. Conversely, if $T$ is a splitting tilting $A$-module and $B=\operatorname{End}_{A}(T)$ is representation-infinite, then so is $A$.

Notation 2.7. Let $A \simeq k Q_{A} / I_{A}$ be a representation-finite algebra. We denote by $\left(R_{A}\right)_{0}$ the subset of $\left(Q_{A}\right)_{0}$ of all the vertices that determine the nilpotency index $r_{A}$ of $\Re(\bmod A)$, that is, $\left(R_{A}\right)_{0}=\left\{u \in\left(Q_{A}\right)_{0} \mid r_{u}=r_{A}-1\right\} \subset\left(Q_{A}\right)_{0}$ where $r_{u}$ is the length of any path from $P_{u}$ to $I_{u}$ going through $S_{u}$.

We are in position to prove Theorem A.

Theorem 2.8. Let $A \simeq k Q_{A} / I_{A}$ be a representation-finite algebra, $T$ be a separating tilting $A$-module and $B=\operatorname{End}_{A} T$. If $B$ is representation-finite and for some $u \in\left(R_{A}\right)_{0}$ we have that $P_{u} \in$ add $T$ then $r_{A} \leq r_{B}$, where $r_{A}$ and $r_{B}$ are the nilpotency indices of $\Re(\bmod A)$ and $\Re(\bmod B)$, respectively.

Proof. Consider $A \simeq k Q_{A} / I_{A}$ and $B=\operatorname{End}_{A} T$, with $T$ a separating tilting $A$-module that satisfies that $P_{u} \in \operatorname{add} T$, for some $u \in\left(R_{A}\right)_{0}$. Assume that $B$ is representation-finite then so is $A$. Let $r_{A}$ and $r_{B}$ be the nilpotency index of $\Re(\bmod A)$ and $\Re(\bmod B)$, respectively.

By hypothesis, the vertex $u \in\left(Q_{A}\right)_{0}$ is such that $r_{u}=r_{A}-1$. Consider a non-zero morphism $\varphi_{u}: P_{u} \rightarrow I_{u}$ that factors trough $S_{u}$, then by Lemma 1.5 we have that $d p\left(\varphi_{u}\right)=r_{A}-1$. Since $T$ is a separating tilting $A$-module and $P_{u}, I_{u} \in \mathcal{T}(T)$, by Proposition 2.1 we have that $0 \neq F\left(\varphi_{u}\right) \in \Re_{B}^{r_{A}-1}\left(F\left(P_{u}\right), F\left(I_{u}\right)\right)$, where $F=\operatorname{Hom}_{A}(T,-)$. Since $\Re_{B}^{r_{A}-1}(\bmod B) \neq 0$, by the maximality of $r_{B}$ we deduce that $r_{A} \leq r_{B}$.

In Theorem 2.8 we compare the nilpotency indices of $\Re(\bmod A)$ and $\Re(\bmod B)$. Now, we are interested to study when such indices coincide.

Let $S$ be a simple $A$-module, $P$ and $I$ be the projective cover and the injective envelope of $S$, respectively. Let $T$ be a tilting module such that $P \in \operatorname{add} T$ and $B=\operatorname{End}_{A} T$. Recall that $P^{\prime}=\operatorname{Hom}_{A}(T, P)$ is an indecomposable projective $B$-module and that $I^{\prime}=\operatorname{Hom}_{A}(T, I)$ is an indecomposable injective $B$-module. Moreover, $P^{\prime} / \operatorname{rad} P^{\prime} \cong \operatorname{soc} I^{\prime}$.

Proposition 2.9. Let $A \simeq k Q_{A} / I_{A}$ be a representation-finite algebra, $T$ be a separating tilting $A$-module and $B=\operatorname{End}_{A} T$. Assume that $B$ is representation-finite algebra and that there exists $u \in\left(R_{A}\right)_{0}$ such that $P_{u} \in$ add $T$, where $P_{u}$ is the projective module corresponding to the vertex $u$. If $r_{A}=r_{B}$, then there exists a non-zero path of irreducible morphisms between indecomposable modules from $F\left(P_{u}\right)$ to $F\left(I_{u}\right)$ of length $r_{A}-1$. 
Proof. Let $A$ be a representation-finite algebra and $T$ be a separating tilting module, with $P_{u} \in \operatorname{add} T$ for some $u \in\left(R_{A}\right)_{0}$. Assume that $B=\operatorname{End}_{A}(T)$ is representation-finite and $r_{A}=r_{B}$. Consider $\varphi_{u}: P_{u} \rightarrow I_{u}$ a non-zero morphism that factors through $S_{u}$. Thus, $d p\left(\varphi_{u}\right)=r_{A}-1$ and there is a non-zero path of irreducible morphisms between indecomposable modules, $P_{u} \stackrel{f_{1}}{\longrightarrow} X_{1} \stackrel{f_{2}}{\longrightarrow} \cdots \stackrel{f_{r_{A}-2}}{\longrightarrow} X_{r_{A}-2} \stackrel{f_{r_{A}-1}}{\longrightarrow} I_{u}$. Since $T$ is a separating tilting module, we claim that for each $i$, the modules $X_{i}$ belong to the subcategory $\mathcal{T}(T)$, because $P_{u} \in \mathcal{T}(T)$ and $\operatorname{Hom}(\mathcal{T}(T), \mathcal{F}(T))=0$. Following Brenner and Butler's theorem, there exists a non-zero path of non-isomorphisms as follows:

$$
\widetilde{\psi}=F\left(P_{u}\right) \stackrel{F\left(f_{1}\right)}{\longrightarrow} F\left(X_{1}\right) \longrightarrow \cdots \longrightarrow F\left(X_{r_{A}-2} \stackrel{F\left(f_{r_{A}}-1\right)}{\longrightarrow} F\left(I_{u}\right) .\right.
$$

It is clear that every $F\left(X_{i}\right)$ is a $B$-module in $\mathcal{Y}(T)$, for $1 \leq i \leq r_{A}-2$. Moreover, each $F\left(f_{i}\right)$ is an irreducible morphism. Indeed, suppose that there exists an integer $j$ with $1 \leq$ $j \leq r_{A}-1$, such that $F\left(f_{j}\right)$ is not irreducible. Then, $F\left(f_{j}\right) \in \Re_{B}^{2}\left(F\left(X_{j-1}\right), F\left(X_{j}\right)\right)$ and hence $\widetilde{\psi} \in \Re^{r_{A}}(\bmod B)=\Re^{r_{B}}(\bmod B)$, a contradiction to the fact that $\Re^{r_{B}}(\bmod B)=0$. Therefore, each morphism $F\left(f_{i}\right)$ is irreducible, proving our result.

The next examples show that the converse of Proposition 2.9 is not necessarily true.

Example 2.10. (a) Consider the algebras $A$ and $B$ given in Example 2.3 (a). Let $T$ be the tilting $A$-module, also given in the same mentioned example. It is not hard to check that the nilpotency index $r_{A}$ of $\Re(\bmod A)$ is four. Moreover, the vertex 3 in $Q_{A}$ belongs to $\left(R_{A}\right)_{0}$ and there exists a non-zero path of irreducible morphisms between indecomposable modules from $P_{3^{\prime}}=\operatorname{Hom}_{A}\left(T, P_{3}\right)$ to $I_{3^{\prime}}=\operatorname{Hom}_{A}\left(T, I_{3}\right)$ of length three. However, the nilpotency index $r_{B}$ of $\Re(\bmod B)$ is five. Therefore, $r_{A} \neq r_{B}$.

(b) If instead of the existence of a vertex $u \in\left(R_{A}\right)_{0}$ such that $P_{u} \in \operatorname{add} T$ where $T$ a separating tilting $A$-module we put the condition that for all $u \in\left(R_{A}\right)_{0}$ we have that $P_{u} \in \operatorname{add} T$ then still the converse of Proposition 2.9 does not hold as we show in our next example.

Let $A$ be the algebra given by the quiver

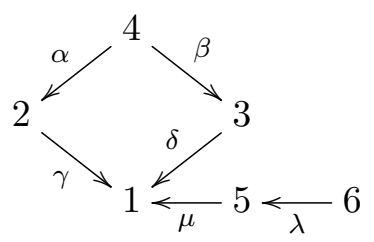

with $I_{A}=<\gamma \alpha-\delta \beta, \mu \lambda>$. The Auslander-Reiten quiver of $\bmod A$ is the following: 


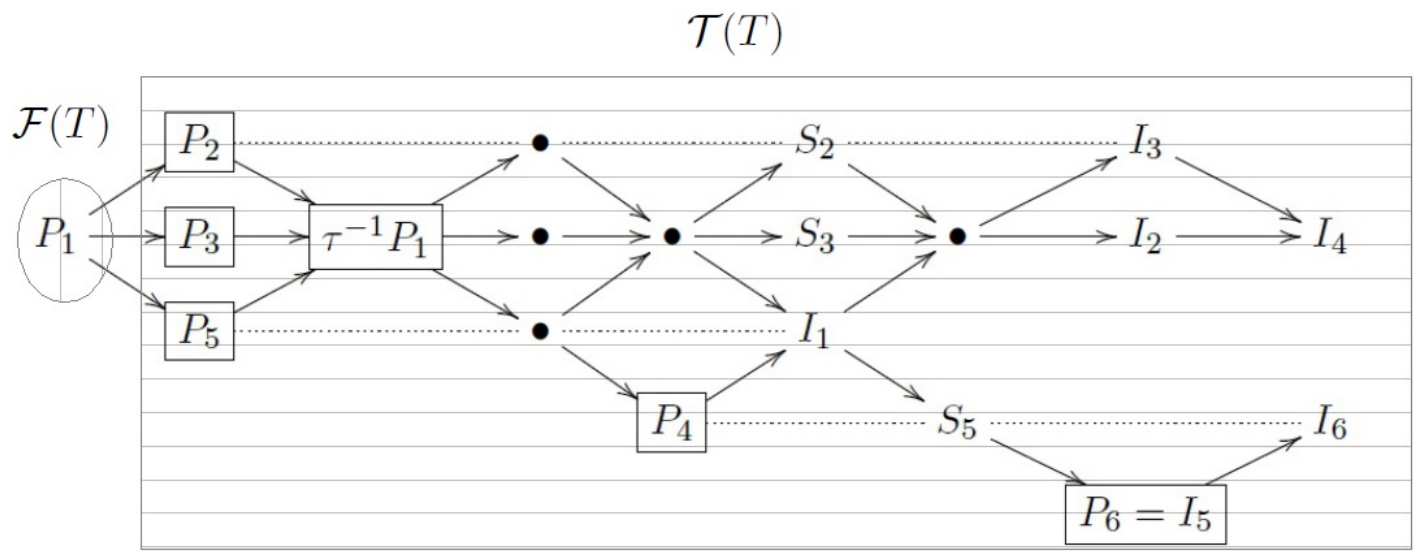

where $T=\tau P_{1} \oplus P_{2} \oplus P_{3} \oplus P_{4} \oplus P_{5} \oplus P_{6}$ is the APR-tilting $A$-module corresponding to the vertex one. The endomorphism algebra $B=\operatorname{End}_{A} T$ is given by the bound quiver:

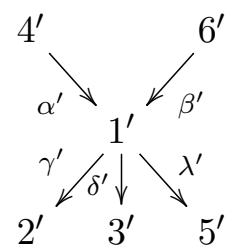

with $I_{B}=<\lambda^{\prime} \alpha^{\prime}, \gamma^{\prime} \beta^{\prime}, \delta^{\prime} \beta^{\prime}>$, and the Auslander-Reiten quiver of $\bmod B$ is the following

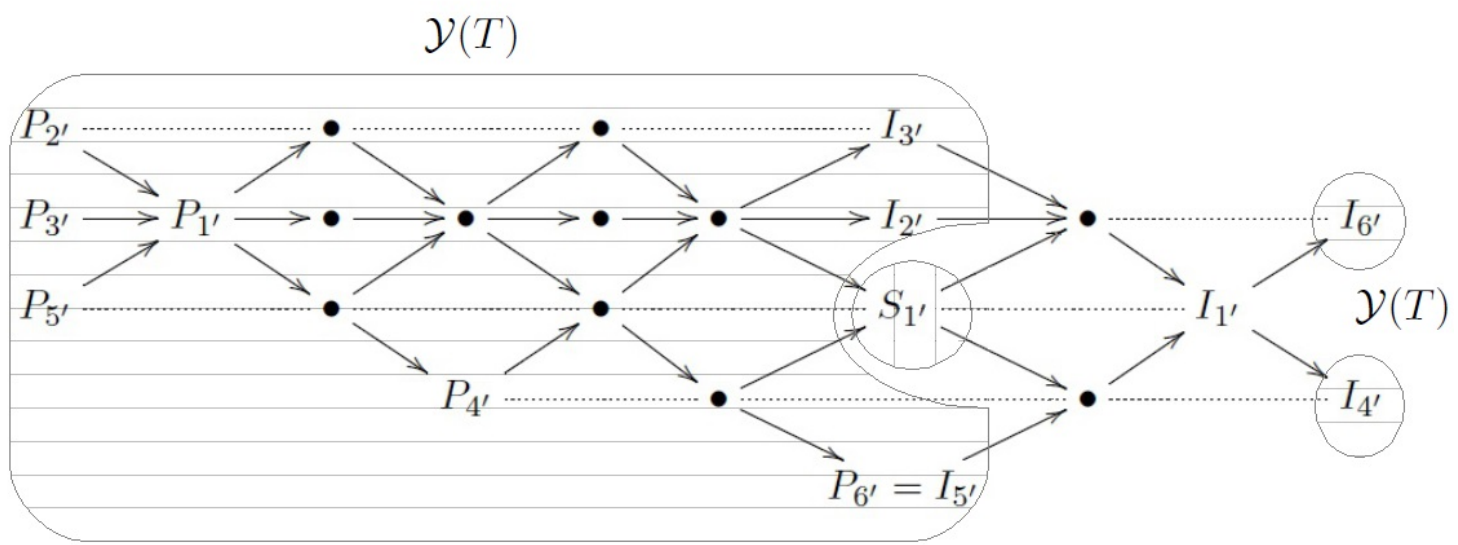

Note that the nilpotency index of $\Re(\bmod A)$ is seven. The set $\left(R_{A}\right)_{0}=\{2,3,5\}$ and the modules $P_{2}, P_{3}$ and $P_{5}$ belong to the subcategory add $T$.

On the other hand, the paths in ind $B$ from $F\left(P_{i}\right)=P_{i^{\prime}}$ to $F\left(I_{i}\right)=I_{i^{\prime}}$ going through the simple $S_{i^{\prime}}$, for $i=2,3,5$, have length six, where $F=\operatorname{Hom}_{A}(T,-)$. However, the nilpotency index of $\Re(\bmod B)$ is eight, and therefore $r_{A} \neq r_{B}$.

In the next section we shall prove that if $B$ is an hereditary algebra, hence $A$ is a tilted algebra, then the converse of the implication in the statement of Proposition 2.9 is still true.

Now, we prove a result useful for further considerations. 
Proposition 2.11. Let $A \simeq k Q_{A} / I_{A}$ be a representation-finite algebra and $P_{a}$ be a simple projective $A$-module. Then, there is a vertex $b \neq a$ such that $r_{a} \leq r_{b}$.

Proof. Since $P_{a}=S_{a}$, then any non-zero morphism $\varphi_{a}: P_{a} \rightarrow I_{a}$ satisfies that $d p\left(\varphi_{a}\right)=r_{a}$. Hence, by [3, Proposition 7.4], there is a non-zero path of irreducible morphisms between indecomposable modules of length $r_{a}$ as follows:

$$
P_{a} \stackrel{h_{1}}{\longrightarrow} X_{1} \stackrel{h_{2}}{\longrightarrow} \cdots \stackrel{h_{r_{a}-1}}{\longrightarrow} X_{r_{a}-1} \stackrel{h_{r_{a}}}{\longrightarrow} I_{a} .
$$

We claim that $X_{1}$ is projective. In fact, otherwise, there is an irreducible morphism $\tau X_{1} \rightarrow P_{a}$. Since $P_{a}$ is projective, then $\tau X_{1}$ is a direct summand of $\operatorname{rad} P_{a}$, but since $P_{a}$ is simple we get to a contradiction. Hence we write $X_{1}=P_{b}$, for some vertex $b \neq a$.

Consider $\psi=h_{r_{a}} \ldots h_{2}: P_{b} \rightarrow I_{a}$. Then, $d p(\psi)=r_{a}-1$. Since $b \neq a$, we have that $\psi$ does not factor through $S_{b}$. Following [8, Lemma 2.2], we know that there is a non-zero morphism $\xi \in \Re_{A}\left(I_{a}, I_{b}\right)$ such that the composition $\xi \psi$ does not vanish and moreover, it factors through $S_{b}$. Therefore, $\xi \psi \in \Re_{A}^{r_{a}}\left(P_{b}, I_{b}\right)$. Finally, by Lemma 1.5 we get that $r_{a} \leq r_{b}$.

A similar result holds if $I_{a}$ is a simple injective $A$-module. Below, we state the result.

Proposition 2.12. Let $A \simeq k Q_{A} / I_{A}$ be a representation-finite algebra. Assume that $Q_{A}$ has at leat one source and that $I_{a}$ is a simple injective $A$-module. Then, there is a vertex $b \neq a$ such that $r_{a} \leq r_{b}$.

Remark 2.13. Let $A \simeq k Q_{A} / I$ be an algebra and $T[a]$ be the APR-tilting module corresponding to a sink $a$ of $Q_{A}$. By the construction of the module $T[a]$ and Proposition 2.11, it follows that there is a vertex $u \in\left(R_{A}\right)_{0}$ such that $P_{u} \in \operatorname{add} T[a]$.

The next lemma is a particular case of Theorem 2.8 .

Lemma 2.14. Let $A \simeq k Q_{A} / I_{A}$ be an algebra and $a \in\left(Q_{A}\right)_{0}$ be a sink. Let $T[a]$ be the $A P R$ tilting $A$-module corresponding to the vertex $a$ and $B=\operatorname{End}_{A}(T[a])$. If $B$ is representationfinite then $r_{A} \leq r_{B}$.

In case that $T[a]$ is an APR-tilting $A$-module, with $a$ a free sink of $Q_{A}$ we prove that the nilpotency indices of $\Re(\bmod A)$ and $\Re(\bmod B)$ coincide. More precisely, Lemma 2.14 and Lemma 2.15 give us Theorem B.

Lemma 2.15. Let $A \simeq k Q_{A} / I_{A}$ be a representation-finite algebra and $a \in\left(Q_{A}\right)_{0}$ a free sink. Let $T[a]$ be the APR-tilting $A$-module corresponding to the vertex $a$ and $B=\operatorname{End}_{A}(T[a])$. Then $r_{A}=r_{B}$.

Proof. Let $A$ be a representation-finite algebra. Since $B=\operatorname{End}_{A}(T[a])$, where $T[a]$ is the APR-tilting $A$-module corresponding to the free sink $a$, then $B$ is also representation-finite.

By Lemma 2.14, we know that $r_{A} \leq r_{B}$. We claim that $r_{A}=r_{B}$. In fact, if $r_{A} \leq r_{B}-1$ then since $a$ is a free sink of $\left(Q_{A}\right)_{0}$, by [1, Lemma p. 178] we know that $a^{\prime}$, the vertex corresponding to $a$ in $Q_{B}$, is a source. Then, by Proposition 2.12, there is a vertex $b^{\prime} \in\left(Q_{B}\right)_{0}$, with $b^{\prime} \neq a^{\prime}$, such that $r_{b^{\prime}}=r_{B}-1$. Consider a non-zero morphism $\widetilde{\varphi}_{b^{\prime}}: P^{\prime}{ }_{b^{\prime}} \rightarrow I^{\prime}{ }_{b^{\prime}}$ that factors through $S^{\prime}{ }_{b^{\prime}}$ in $\bmod B$. Then, by Lemma 1.5 we have that $d p\left(\widetilde{\varphi}_{b^{\prime}}\right)=r_{B}-1$.

Moreover, the indecomposable $B$-modules $P^{\prime}{ }_{b^{\prime}}$ and $I^{\prime}{ }_{b^{\prime}}$ belong to the subcategory $\mathcal{Y}(T[a])$. Then, by Brenner and Butler's theorem, there are indecomposable $A$-modules $M, N \in$ $\mathcal{T}(T[a])$ and a non-zero morphism $f: M \rightarrow N$ such that $P^{\prime}{ }_{b^{\prime}}=F(M), I^{\prime}{ }_{b^{\prime}}=F(N)$ and $\widetilde{\varphi}_{b^{\prime}}=F(f)$. By Corollary 2.4, we know that $d p(f)=r_{B}-1$, a contradiction to the fact that $\Re^{r_{A}}(\bmod A)=0$ and $r_{A} \leq r_{B}-1$. Therefore, $r_{A}=r_{B}$. 
As an immediate consequence of Proposition 2.11 and Proposition 2.12, we know that to compute the nilpotency index of the radical of a module category of a representation-finite algebra, it is enough to analyze the vertices of $Q_{A}$ which are neither sinks nor sources. Below we state such a result.

Theorem 2.16. Let $A=k Q_{A} / I_{A}$ be a representation-finite algebra. Consider $\mathcal{V}$ the subset of vertices of $Q_{A}$ which are neither sinks nor sources. Assume that $\mathcal{V} \neq \emptyset$ and that for every $a \in \mathcal{V}$, we write $r_{a}=d_{r}\left(\iota_{a}\right)+d_{l}\left(\theta_{a}\right)$, where $\iota_{a}: \operatorname{rad} P_{a} \rightarrow P_{a}$ and $\theta_{a}: I_{a} \rightarrow I_{a} / \operatorname{soc} I_{a}$ are irreducible morphisms, with $P_{a}$ and $I_{a}$ the projective and the injective modules corresponding to the vertex a. Then, $r_{A}=\max \left\{r_{a}\right.$, con $\left.a \in \mathcal{V}\right\}+1$ is the nilpotency index of $\Re(\bmod A)$.

Remark 2.17. If the set $\mathcal{V}$ is empty, that is, all the vertices of $Q_{A}$ are sinks or sources, then the algebra is hereditary. In the next section, we study the nilpotency index of the radical of the module category of a representation-finite hereditary algebra, see Theorem 3.3 .

\section{Application to iterated tilted algebras}

The aim of this section is to study a bound for the nilpotency index of the radical of the module category of an iterated tilted algebra of Dynkin type.

We start proving some essential results for our further considerations.

Proposition 3.1. Let $H$ be a representation-finite hereditary algebra. Then, $\Gamma_{H}$ is a component with length.

Proof. In [6, Section 4.3], it was proved that the orbit graph $O\left(\Gamma_{H}\right)$ is a deformation retract of $\Gamma_{H}$. Therefore, $O\left(\Gamma_{H}\right)$ is a tree and consequently $\Gamma_{H}$ is a simply connected translation quiver. Hence, $\Gamma_{H}$ is a component with length.

Lemma 3.2. Let $H \simeq k Q_{H}$ be a representation-finite hereditary algebra. Then, $r_{a}=r_{b}$, for all vertices $a, b \in\left(Q_{H}\right)_{0}$.

Proof. Let $a \in\left(Q_{H}\right)_{0}$. We know that $r_{a}$ is the length of a path of irreducible morphisms between indecomposable modules from the projective $P_{a}$ to the injective $I_{a}$, going through the simple $S_{a}$. Since $\Gamma_{\bmod H}$ is with length, it is enough to analyze any path from $P_{a}$ to $I_{a}$.

Let $i, j \in\left(Q_{H}\right)_{0}$ be vertices such that there is an arrow from $i$ to $j$. Then, there are irreducible morphisms $P_{j} \rightarrow P_{i}$ and $I_{j} \rightarrow I_{i}$, where $P_{j}$ and $P_{i}$ are the projective $H$-modules corresponding to the vertices $j$ and $i$ respectively; and $I_{j}$ and $I_{i}$ are the injective $H$-modules corresponding to the vertices $j$ and $i$, respectively. Moreover, by [8, Lemma 2.3], there is a non-zero path $P_{a} \rightsquigarrow I_{a}$, for all $a \in\left(Q_{H}\right)_{0}$. Then, in $\Gamma_{\bmod H}$ we have the following two paths of irreducible morphisms:

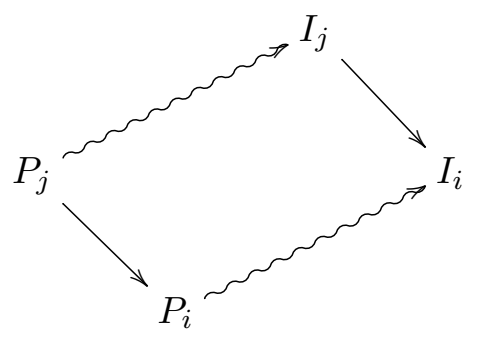

Assume that the path $P_{j} \rightsquigarrow I_{j}$ has length $m$. Then, the path $P_{j} \rightsquigarrow I_{j} \rightarrow I_{i}$ has length $m+1$, because the arrow denote an irreducible morphism between indecomposable modules. Since $\Gamma_{\bmod H}$ is with length, the path $P_{j} \rightarrow P_{i} \rightsquigarrow I_{i}$ has also length $m+1$ and hence, the 
path $P_{i} \rightsquigarrow I_{i}$ has length $m$. Since $H$ is connected, then all paths of the form $P_{a} \rightsquigarrow I_{a}$, with $a \in\left(Q_{H}\right)_{0}$ have length $m$, and therefore $r_{a}=r_{b}$ for all $a, b \in\left(Q_{H}\right)_{0}$.

By [18, Theorem 4.11], we recall the following result for a representation-finite hereditary algebra.

Theorem 3.3. Let $H=k Q$ be a representation-finite hereditary algebra and let $r_{H}$ be the nilpotency index of $\Re(\bmod H)$.

(a) If $\bar{Q}=A_{n}$, then $r_{H}=n$, for $n \geq 1$.

(b) If $\bar{Q}=D_{n}$, then $r_{H}=2 n-3$, for $n \geq 4$.

(c) If $\bar{Q}=E_{6}$, then $r_{H}=11$.

(d) If $\bar{Q}=E_{7}$, then $r_{H}=17$.

(e) If $\bar{Q}=E_{8}$, then $r_{H}=29$.

Now, we prove a necessity condition of Proposition 2.9, when $B$ is a hereditary algebra.

Proposition 3.4. Let $A \simeq k Q_{A} / I_{A}$ be an algebra and $B=\operatorname{End}_{A} T$ be a representation-finite hereditary algebra, with $T$ a tilting A-module. Assume that there exists a vertex $u \in\left(R_{A}\right)_{0}$ such that $P_{u} \in \operatorname{add} T$. Then, $r_{A}=r_{B}$ if and only if there is a nonzero path of irreducible morphisms $F\left(P_{u}\right) \rightarrow X^{\prime}{ }_{1} \rightarrow \ldots \rightarrow X^{\prime}{ }_{r_{A}-2} \rightarrow F\left(I_{u}\right)$ of length $r_{A}-1$.

Proof. Since $B$ is a representation-finite hereditary algebra, then $T$ is a separating tilting $A$-module and $A$ is also a representation-finite algebra. Consider $r_{A}$ and $r_{B}$ the nilpotency indices of $\Re(\bmod A)$ and $\Re(\bmod B)$, respectively. Assume that there exists a vertex $u \in\left(R_{A}\right)_{0}$ such that $P_{u} \in \operatorname{add} T$. The sufficient condition follows from Proposition 2.9 .

Conversely, by hypothesis there exists in $\bmod B$ a nonzero path of irreducible morphisms $F\left(P_{u}\right) \rightarrow X^{\prime}{ }_{1} \rightarrow \ldots \rightarrow X^{\prime}{ }_{r_{A}-2} \rightarrow F\left(I_{u}\right)$ of length $r_{A}-1$. We shall prove that $r_{A}=r_{B}$. We know that $F\left(P_{u}\right)=P^{\prime} u^{*}$ and $F\left(I_{u}\right)=I^{\prime} u^{*}$ are the projective and the injective $B$-modules corresponding to the vertex $u^{*} \in\left(Q_{B}\right)_{0}$.

On the other hand, since $B$ is an hereditary algebra, by Lemma 3.2 we have that any path of irreducible morphism from a projective to the injective corresponding to the same vertex has length $r_{B}-1$. In particular, the above path has length $r_{B}-1$. Therefore, $r_{A}=r_{B}$.

The following result was proved by Happel in [14.

Theorem 3.5. [14, Teorema 6.2] Let $\Delta$ be a Dynkin quiver and $A$ be an iterated tilted algebra of type $\Delta$. Then, there exist a sequence of algebras $A=A_{0}, A_{1}, \ldots, A_{m}=k \Delta$ and a sequence of APR-tilting $A_{i}$-modules $T^{(i)}$, for $0 \leq i<m$, such that $A_{i+1}=\operatorname{End}_{A_{i}} T^{(i)}$.

As a consequence of Lemma 2.14 and Theorem 3.3 we get an upper bound for the nilpotecy index of an iterated tilted algebra.

Corollary 3.6. Let $\Delta$ be a Dynkin quiver and $A$ be an iterated tilted algebra of type $\Delta$. Consider $r_{A}$ the nilpotency index of $\Re_{A}(\bmod A)$.

(a) If $\bar{\Delta}=A_{n}$, then $r_{A} \leq n$ for $n \geq 1$.

(b) If $\bar{\Delta}=D_{n}$, then $r_{A} \leq 2 n-3$ for $n \geq 4$.

(c) If $\bar{\Delta}=E_{6}$, then $r_{A} \leq 11$.

(d) If $\bar{\Delta}=E_{7}$, then $r_{A} \leq 17$.

(e) If $\bar{\Delta}=E_{8}$, then $r_{A} \leq 29$.

The next example shows that there exists iterated tilted algebras such that the nilpotency index of the radical of its module category have the maximum value of the bound given in Corollary 3.6. Moreover, two iterated tilted algebras of the same Dynkin type, may have different nilpotency indices. 
Example 3.7. Let $A_{1}$ be the algebra given by the bound quiver

$$
1 \stackrel{\alpha, \ldots \ldots \ldots \beta}{\longrightarrow} 2 \stackrel{\gamma}{\longrightarrow} 3 \stackrel{\delta}{\longrightarrow} 5
$$

with $I_{1}=<\beta \alpha>$ and $A_{2}$ be the algebra given by the bound quiver

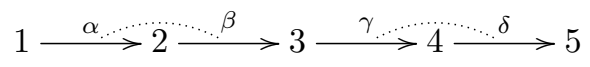

with $I_{2}=<\beta \alpha, \delta \gamma>$.

Observe that both algebras are iterated tilted algebras of type $A_{5}$. By Corollary 3.6, we know that $r_{A_{i}} \leq 5$ for $i=1,2$. Computing each nilpotency index, we get that $r_{A_{1}}=5$ but $r_{A_{2}}=4$.

An algebra $A$ is called a directed algebra if each indecomposable A-module is directed, that is, it does not belong to any cycle in $\bmod A$.

Proposition 3.8. [14, Corollary 3.6] Let $A$ be a representation-finite iterated tilted algebra. Then, $A$ is a directed algebra.

Recall that an algebra $A$ is standard if the category ind $A$ is equivalent to the mesh category of $\Gamma_{A}$, see [13, 2.5]. It is known by [7] that a directed algebra is standard.

Following [13, by a bypass of an irreducible morphism $f: X \rightarrow Y$, with $X$ and $Y$ indecomposable modules, we mean a path $X \stackrel{t_{1}}{\longrightarrow} Y_{1} \stackrel{t_{2}}{\longrightarrow} Y_{2} \longrightarrow \cdots \longrightarrow Y_{n} \stackrel{t_{n+1}}{\longrightarrow} Y$ in ind $A$ of length $n+1 \geq 2$, where $t_{1}$ and $t_{n+1}$ are irreducible morphisms, $X ¥ Y_{n}$ and $Y \not Y_{1}$.

In [13, Corollary 2.9], the authors proved that if we consider a standard algebra $A$ and a

nonzero composition of $n$ irreducible morphisms between indecomposable modules in $\Re_{A}^{n+1}$, then there exists a bypass or a cycle in $\Gamma_{A}$. Since, by [17, there are no bypasses in a directed algebra, then we have the following result.

Lemma 3.9. [13, Corollary 2.11] Let $A$ be a directed algebra. Let $h_{i}: X_{i} \rightarrow X_{i+1}$ be irreducible morphisms between indecomposable modules, for $1 \leq i \leq n$. Then $h_{n} \ldots h_{1} \in$ $\Re_{A}^{n+1}\left(X_{1}, X_{n+1}\right)$ if and only if $h_{n} \ldots h_{1}=0$.

Our next result is an immediately consequence of Proposition 3.8 and Lemma 3.9

Proposition 3.10. Let $\Delta$ be a Dynkin quiver and $A$ be an iterated tilted algebra of type $\Delta$. Let $h_{i}: X_{i} \rightarrow X_{i+1}$ be irreducible morphisms between indecomposable modules, for $1 \leq i \leq n$. Then $h_{n} \ldots h_{1} \in \Re_{A}^{n+1}\left(X_{1}, X_{n+1}\right)$ if and only if $h_{n} \ldots h_{1}=0$.

\section{REFERENCES}

[1] I. Assem. Separating splitting tilting modules and hereditary algebras. Canadian Mathematical Bulletin, Vol. 30, 2, (1987), 177-181.

[2] I. Assem, D. Simson, A. Skowronski. Elements of the representation theory of associative algebras. London Mathematical Society, Student Texts 65, Cambridge University Press, (2006).

[3] M. Auslander, I. Reiten, S. O. Smal $\phi$. Representation theory of artin algebras. Cambridge Studies in Advanced Mathematics 36, Cambridge University Press, (1995).

[4] R. Bautista. Irreducible morphisms and the radical of a category. An. Inst. Mat. Nac. Autónoma México 22, (1982), 83-135.

[5] S. Brenner, M. C. R. Butler. Generalization of the Bernstein-Gelfand-Ponomarev reflection functors. Lecture Notes in Math., 832, Springer-Verlag, Berlin-Heidelberg, New York, (1980), 103-169.

[6] K. Bongartz, P. Gabriel. Covering Spaces in Representation-Theory. Inventiones Mathematicae 65, Springer Verlag, (1982), 331-378.

[7] O. Bretscher, P. Gabriel. The standard form of a representation-finite algebra. Bulletin Soc. Math. France 111, (1983), 21-40. 
[8] C. Chaio. On the Harada and Sai Bound. Bulletin of the London Mathematical Society 44, 6, (2012), 1237-1245.

[9] C. Chaio. Problems solved by using degrees of irreducible morphisms. Contemporary Mathematics 607, American Math. Soc, (2014), 179-203.

[10] C. Chaio, P. Le Meur, S. Trepode. Degrees of irreducible morphisms and finite-representation type. Journal of London Mathematical Society II 84, 1, (2011), 35-57.

[11] C. Chaio, S. Liu. A note on the radical of a module category. Communications in Algebra 41, 12, (2013), 4419-4424.

[12] C. Chaio, M.I. Platzeck, S. Trepode. On the degree of irreducible morphisms. Journal of Algebra 281, 1, (2004), 200-224.

[13] C. Chaio, S. Trepode. The composite of irreducible morphisms in standard components. Journal of Algebra 323, 4, (2010), 1000-1011.

[14] D. Happel. Triangulated categories in the representation theory of finite-dimensional algebras. London Mathematical Society, Lecture Note Series 119, Cambridge University Press, (1987).

[15] D. Happel, C.M. Ringel. Tilted algebras. Trans. American Mathematical Society 284, (1982), 399443.

[16] S. Liu. Degree of irreducible maps and the shapes of Auslander-Reiten quivers. Journal of London Mathematical Society 2, 45, (1992), 32-54.

[17] L. Salmeron. Stratifcation of fnite Auslander-Reiten quivers without oriented cycles. Journal of London Mathematical Society s2, 31, (1985), 224-230.

[18] D. Zacharia. The preprojective partition for Hereditary artin algebras. Transactions of the American Mathematical Society 274, 1, (1982), 327-343.

Centro marplatense de Investigaciones Matemáticas, Facultad de Ciencias Exactas y Naturales, Funes 3350, Universidad Nacional de Mar del Plata, 7600 Mar del Plata, Argentina

E-mail address: claudia.chaio@gmail.com

Centro marplatense de Investigaciones Matemáticas, Facultad de Ciencias Exactas y NatuRales, Funes 3350, Universidad Nacional de Mar del Plata, 7600 Mar del Plata, Argentina

E-mail address: victoria.guazzelli@hotmail.com 